\title{
Classical Hodgkin's Lymphoma with Skeletal Muscle Involvement: A Testing Case
}

\author{
Syed Ali Shazif Baqari1, Mairah Razi2 ${ }^{2}$ Javeria Hayat ${ }^{3}$ and Rabia Mohammad Wali1
}

\begin{abstract}
Skeletal muscle involvement in Hodgkin lymphoma is very rare. An 11-year male child presented with stage IV Hodgkin lymphoma and skeletal muscle involvement in right gluteal, piriformis and psoas muscles. He had resistant disease. He achieved remission with 3rd line chemotherapy, EPIC; and successfully underwent high dose chemotherapy with autologous stem cell rescue. The patient is well after 15 months of follow-up. Successful treatment signifies positron emission computed tomography (PET/CT) evaluation and multidisciplinary team discussions in diagnosis and management of a rare Hodgkin lymphoma presentation.
\end{abstract}

Key Words: Hodgkin lymphoma, Positron emission computed tomography, Skeletal muscle.

\section{INTRODUCTION}

Hodgkin's lymphoma $(\mathrm{HL})$ is a malignant condition of lymphoid system which commonly involves supradiaphragmatic recesses and spreads from one nodal group contiguously to the other. The skeletal muscle involvement is a very rare manifestation of $\mathrm{HL}$ and indicates extensive disease spread beyond the usual organs' involvement.

About $15 \%$ cases present with extra-nodal invasion of adjacent tissue and $5-10 \%$ with hematogenous spread. 1,2 The incidence of primary lymphomatous skeletal muscle involvement in $\mathrm{HL}$ is $0.3 \%{ }^{3}$

We describe a rare presentation of a rather common lymphoma, its histopathological findings and important radiologic features.

\section{CASE REPORT}

An 11-year boy presented with generalised lymph node enlargement for one year with fever, weight loss and cough for six months. The nodes of neck, armpits and groins progressively enlarged without pain, redness or ulcer formation. Patient received multiple blood transfusions from different hospitals. There was no family history of any malignancy. Systemic review revealed malaise, fatigue, skin infections and joint pains.

On clinical examination, he was having pallor, bilateral malar rash and soft tender abdomen with palpable liver

1 Department of Pediatric Oncology / Neclear Medicine ${ }^{2}$, Shaukat Khanum Memorial Cancer Hospital and Research Centre, Lahore, Pakistan

3 Medical Student, Dow Medical College, Karachi, Pakistan Correspondence: Dr. Syed Ali. Shazif Baqari, Department of Pediatric Oncology, Shaukat Khanum Memorial Cancer

Hospital and Research Centre, Lahore, Pakistan

E-mail: alishazif@hotmail.com

Received: May 02, 2018; Accepted: August 02, 2018 and spleen 3 and $4 \mathrm{~cm}$ below right and left costal margins, respectively.

On blood workup, complete blood counts showed bicytopenia with leukocytosis and normal renal and liver function tests. Renogram showed poor function of the right kidney but normal left kidney.

Diagnosis was made on histopathology of excised cervical node which confirmed the presence of Reed Sternberg (RS) cells. RS cells, found characteristically in $\mathrm{HL}$, are morphologically distinct cells and are mandatory for diagnosis (Figure 1). ${ }^{4}$ Further, immunohistochemistry (IHC) panel revealed positive CD30, CD15, and PAX5 in large cells. Bone marrow aspiration and biopsy showed bilateral bone marrow involvement. Baseline 18F-FDG $\mathrm{PET} / \mathrm{CT}$ demonstrated hypermetabolic lymph nodes above and below the diaphragm with lung, renal and musculoskeletal involvement. There was increase in bulk of right gluteal, piriformis and psoas muscles with internal necrotic changes and abnormal metabolic activity. An abnormal metabolic activity with underlying scattered sclerotic foci was noted throughout axial and appendicular skeleton, consistent with osseous involvement as shown in Figure 2. Disease was staged as stage IV according to Ann Arbor staging system. ${ }^{5}$

We also performed the biopsy of right gluteal mass as it was unusual for $\mathrm{HL}$ to involve this tissue. On histopathology, as shown in Figure 1, there were scattered large atypical cells with large nuclei and prominent nucleoli in background of mixture of lymphocytes, plasma cells and eosinophils. On immunohistochemistry (IHC) CD30, CD15, and PAX5 were positive, but CD20 and leukocyte common antigen (LAC) were negative in large cells.

We started his chemotherapy as CODPac (Cyclophosphamide, Vincristine, Prednisone, Dacarbazine) and ABVD (Adriamycin, Bleomycin, Vinblastine, Dacarbazine) 


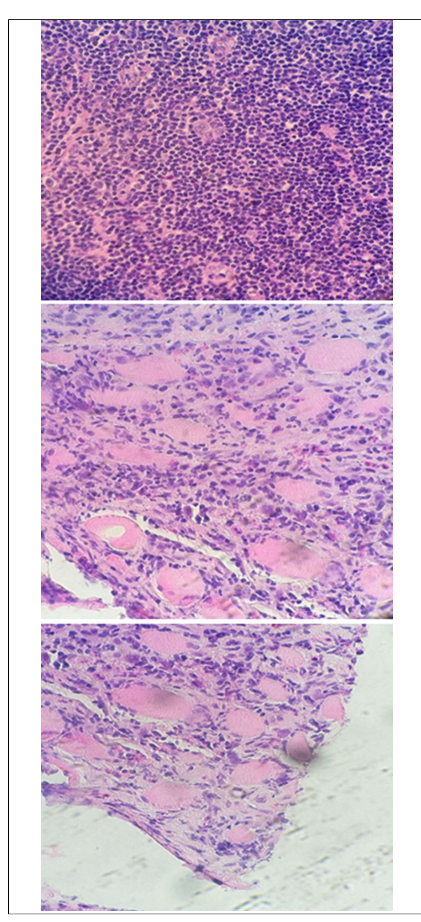

Figure 1: Histopathology images showing muscle bundles infiltrated by lymphoma cell.

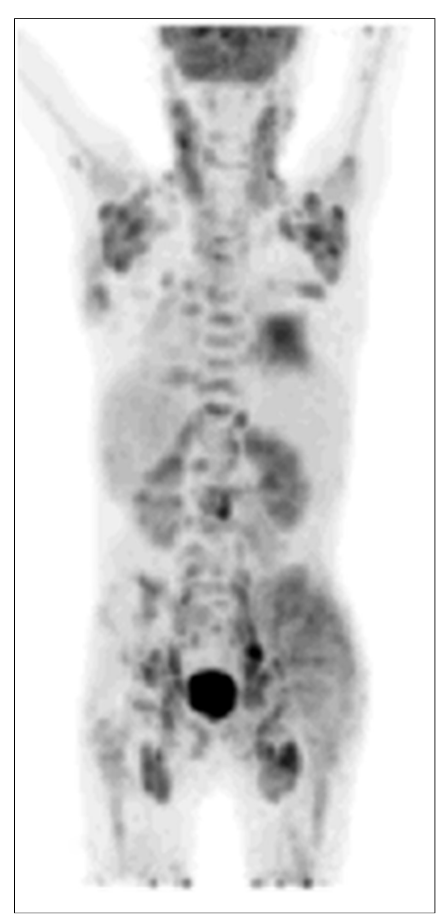

Figure 2 (A): PET/CT image. Coronal maximum intensity projection (MIP) shows extensive hypermetabolic nodal disease above and below the diaphragm with pulmonary, renal, osseous, and muscular involvement.
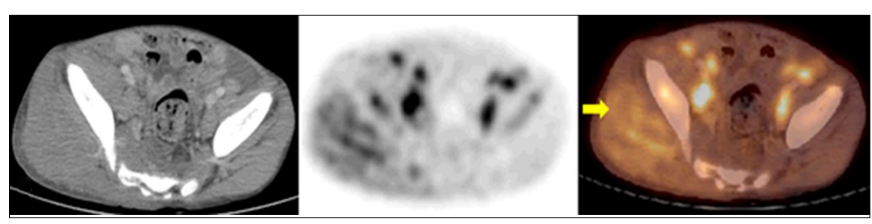

Figure 2 (B): Axial images of CT, PET and fusion PET/CT images show abnormal uptake in right gluteal muscle which appears bulky, consistent with disease involvement.

according to our institutional protocol. After two cycles, interim PET/CT scan showed partial morphologic and metabolic response with residual nodal disease above and below the diaphragm and residual osseous lesions. We observed complete resolution of muscular and renal disease and non-avidity of pulmonary nodules. However, disease progression was observed in right inguinal lymph node. Excisional biopsy of right inguinal lymph node confirmed disease progression.

We started second line chemotherapy with Gemcitabine and Vinorelbine. After two cycles, PET/CT scan showed new abnormal metabolic activity in previously non-avid left external iliac as well as right inguinal nodes. Excisional biopsy of right inguinal node was once again positive for lymphomatous involvement. We commenced with third line chemotherapy, EPIC (Etoposide, Prednisolone, Ifosfamide and Cisplatin). This time interim PET/CT scan showed good partial response with regression of cervical, axillary, right inguinal and left external iliac lymph nodes.
Based on PET/CT findings, multidisciplinary team decided to perform bone marrow harvesting with EPIC 3 , and planned high dose chemotherapy with autologous stem cell rescue. We failed to harvest after EPIC 3, so EPIC 4 was given and harvesting procedure was completed. The day 10 cisplatin was substituted with high dose cyclophosphamide for harvesting in EPIC 4. We collected 4.6 million stem cells through apheresis. Patient successfully underwent BEAM (BiCNUcarmustine, Etoposide, Ara-C-cytarabine, Melphalan) chemotherapy followed by autologous stem cell rescue. Engraftment occurred on day +13 and we observed no significant complication.

Patient is currently 15 months post-treatment and was well on his last follow-up visit. Follow-up assessment was done with clinical examination, ultrasonography abdomen, X-ray chest, complete blood counts, and erythrocyte sedimentation rate (ESR).

\section{DISCUSSION}

Musculoskeletal involvement in malignant lymphomas occurs due to dissemination of primary disease. Skeletal muscle is a very rare site for extra-nodal involvement; relatively common sites are head, neck, skin and gastrointestinal tract.

It is relatively more common in non-Hodgkin's lymphoma $(\mathrm{NHL})$. Although we do find detailed features of skeletal involvement in $\mathrm{HL}$ in textbooks, published literature on uncommon manifestations of $\mathrm{HL}$ such as lymphomatous soft tissue masses is insufficient.

Role of F18-fluorodeoxyglucose (FDG) positron emission tomography (PET) has been established since last decade for accurate delineation of disease extent in HL. 6 Asymmetrical uptake or multifocal accumulation of F18FDG in muscles can raise the suspicion of lymphomaous involvement of musculoskeletal system. ${ }^{7}$ In this case, PET/CT scan was used as the imaging modality owing to its high sensitivity and being a standard modality for staging.

Most common lymph nodes involved in HL are cervical, axillary and inguinal. In our patient, all of the aforementioned groups were palpable and a diagnosis of $\mathrm{HL}$ was conveniently made through cervical node biopsy. The characteristic large cells called RS cells stained positively for CD30, CD15 and PAX5 on IHL.

Baseline PET/CT scan highlighted hypermetabolic disease involvement of gluteal, psoas and piriformis muscles. This was confirmed as extra-nodal lymphomatous involvement on histopathology. It was noted that the RS cells stained positive for CD30 and PAX5. CD30 is a marker for $\mathrm{HL}$; whereas, PAX5 is a transcription factor that is expressed in both $\mathrm{HL}$ and B-cell NHL. Furthermore, large cells stained negative for CD20, which is a B-cell marker, ruling out B-cell NHL. Large cells were also stained negative for LCA, a valuable 
marker which labels lymphoma and leukemia of $B$ and T-cell origin.

Multidisciplinary discussions are important for devising a diagnostic strategy and establishing a treatment plan for such patients. Progression of disease after first and second line chemotherapy indicated aggressive nature of the disease. Although patient is still in remission more than 1 year after autologous stem cell rescue, close regular follow-ups and evaluations are required. As disease is resistant in nature, it holds a high risk of recurrence.

We believe that successful treatment of this case is an important landmark to diagnostic and management approach for an unusual presentation of a malignant lymphoma. Moreover, it emphasises the significance of $\mathrm{PET} / \mathrm{CT}$ scan and multidisciplinary discussions in diagnosing and managing such a rare case, where available clinical guidelines were limited.

\section{REFERENCES}

1. Guermazi A, Ooi CGG. Extranodal Hodgkin disease. In: Radiological imaging in hematological malignancies. Medical radiology (diagnostic imaging) Springer, Berlin, Heidelberg 2004.

2. Komatsuda M, Nagao T, Arimori S. An autopsy case of malignant lymphoma associated with remarkable infiltration in skeletal muscles. Rinsho Ketsueki 1981; 22:891-5.

3. Suresh S, Saifuddin A, O'Donnel P. Lymphoma presenting as a musculoskeletal soft tissue mass: MRI findings in 24 cases. Eur Radiol 2008; 18:2628-34.

4. Gopas J, Stern E, Zurgil U, Ozer J, Ben-Ari A, Shubinsky G, et al. Reed-sternberg cells in Hodgkin's lymphoma present features of cellular senescence. Cell Death Dis 2016; 7:e2457.

5. Donaldson SS. Introduction and historical background: Pediatric Hodgkin lymphoma. Pediatr Lymphoma 2007; 1-5.

6. Burton $\mathrm{C}$, Ell P, Linch D. The role of PET imaging in lymphoma. Br J Haematol 2004; 126:772-84.

7. Paes FM, Kalkanis DG, Sideras PA, Serafini AN. FDG PET/CT of extranodal involvement in non-Hodgkin lymphoma and Hodgkin disease. Radiographics 2010; 30:269-91. 